\title{
Genetic, clinical, and laboratory markers for DOCK8 immunodeficiency syndrome
}

\author{
Qian Zhang ${ }^{\mathrm{a}}$, Jeremiah C. Davis ${ }^{\mathrm{b}}$, Christopher G. Dove ${ }^{\mathrm{a}}$ and Helen C. Su ${ }^{\mathrm{a}, *}$ \\ ${ }^{a}$ Laboratory of Host Defenses, National Institute of Allergy and Infectious Diseases, National Institutes of Health, \\ Bethesda, MD, USA \\ ${ }^{\mathrm{b}}$ Seattle Children's Hospital/University of Washington School of Medicine, Department of Pediatrics, Seattle, USA
}

\begin{abstract}
DOCK8 immunodeficiency syndrome (DIDS) is a combined immunodeficiency characterized by recurrent viral infections, severe atopy, and early onset malignancy. Genetic studies revealed large, unique deletions in patients from different families and ethnic backgrounds. Clinical markers of DIDS include atopic dermatitis, allergies, cutaneous viral infections, recurrent respiratory tract infections, and malignancy. Immune assessments showed T cell lymphopenia, hyper-IgE, hypo-IgM, and eosinophilia. The impaired lymphocyte functions in DIDS patients appear central for disease pathogenesis.
\end{abstract}

Keywords: DOCK8, DIDS, combined immunodeficiency, hyper-IgE syndrome, atopic dermatitis, cutaneous viral infections, lymphopenia

\section{Introduction}

DOCK8 immunodeficiency syndrome (DIDS) is a combined immunodeficiency characterized by recurrent viral infections, severe atopy, and early onset malignancy [1]. Some patients were previously diagnosed with an autosomal recessive form of hyperIgE syndrome (AR-HIES). Our discovery that DOCK8 loss-of-function mutations are responsible for this disease [1] was confirmed by another group in additional patients [2]. Two recently generated mouse models having Dock8-deficiency also demonstrate similar immunologic phenotypes [3]. In humans, large genomic deletions are the most common mutation, and require newer genetic approaches for identification (Table 1) [1, 2]. Clinical features of DIDS are helpful in screening patients, yet are not conclusive. Here we review the current knowledge of DOCK8 and discuss how work in related conditions in humans and mice provides insight into this fascinating disease.

* Corresponding author: Dr. Helen Su, Building 10CRC, Room 5W3940, 10CRC Center Dr., MSC 1456, Bethesda, MD 20892-1456, USA. Fax: +1 301480 0983; E-mail: hsu@niaid.nih.gov.
DOCK8 (Dedicator of cytokinesis 8) belongs to the DOCK180 family, which contain two domains: DHR1 and DHR2 (Dock homology regions 1 and 2) [4,5]. The family is composed of 11 atypical guanine exchange factors (GEFs), which activate Rho-GTPases including RAC1, RAC2, and CDC42 [6-8]. These GTPases are important in regulating many cellular functions including actin cytoskeletal organization, cell-cycle progression, and gene expression [9-12].

DOCK8 belongs to the DOCK-C subfamily of the DOCK180 family, which can activate RAC and CDC42 $[6,8,13,14]$. DOCK8 was shown in a yeast 2-hybrid assay to interact with either RAC1 or CDC42 [15]. Despite being ubiquitously expressed in tissues, RAC1 is not highly expressed in lymphocytes (http://bioinfo.wilmer.jhu.edu/tiger). By contrast, DOCK8 is selectively expressed in the immune system, suggesting that in lymphocytes DOCK8 primarily acts through $\mathrm{CDC} 42$ rather than RAC1. The other two members of the DOCK-C subfamily are exclusively expressed in the nervous system and function to direct neuronal cell growth $[8,16]$. At the time of our discovery, the only member of the DOCK180 family having known immune system functions was Dock2. Mice deficient in Dock2 display impairments in neu- 
trophil chemotaxis and $\mathrm{T}$ and $\mathrm{B}$ cell homing to lymph nodes. $\mathrm{T}$ cell receptor (TCR) translocation towards the antigen presenting cell (APC) during immunological synapse formation is also impaired, which results in reduced $\mathrm{T}$ cell proliferation after antigen stimulation [17-19]. Although the Dock2-deficient mice have been well studied, no human disease has yet been associated with DOCK2 mutations. DIDS is the first human disease resulting from mutations in a DOCK180 family protein, and as such illuminates novel functions of DOCK8 within the immune system.

\section{Genetic markers of DOCK8 immunodeficiency syndrome}

\subsection{Large deletions associated with CNVs are frequent in DIDS}

DOCK8 spans almost $250 \mathrm{~kb}$ of genomic DNA on the short arm of chromosome 9 just proximal to the telomere. The longest reference mRNA sequence for $D O C K 8$ in the National Center for Biotechnology Information (NCBI) database (NM_203447.3) contains 48 coding exons. However, 5'-rapid amplification of cDNA ends (RACE) showed that exon 1 was not transcribed in primary $\mathrm{T}$ cells (H. Jing and H. Su, unpublished data). Moreover, our cDNA sequencing also demonstrated expression in lymphocytes of all remaining exons, consistent with isoform 3 (NM_001193536.1).

DIDS is an autosomal recessive disease caused by loss-of-function mutations in the DOCK8 gene [1]. Most cases are associated with large deletions that lead to absent or trace amounts of expressed DOCK8 protein (Table 1) [1,2]. To date, a total of 27 families have been published [1,2]. Of these, six families reported by Engelhardt et al. were characterized by clinical phenotype and lacked mutational confirmation on both alleles [2]. Among the 21 families having identified mutations, all mutations were unique to each family. The types of mutations so far identified are summarized in Table 1. Fourteen families $(66 \%)$ possess deletions that affect one or more exons. Large homozygous deletions were the most common genotype in patients due to the number of consanguineous families studied, whereas compound heterozygous mutations that include large deletions in one allele were observed in outbred families [1, 2].

The high frequency of large deletions seen in DIDS reflects copy number variations $(\mathrm{CNV})$ inherent to this
Table 1

Classification of $D O C K 8$ mutations in DIDS

\begin{tabular}{lc}
\hline Mutation type & Number of families \\
\hline $\begin{array}{l}\text { Large homozygous or compound } \\
\text { heterozygous deletion }\end{array}$ & $10(48 \%)$ \\
$\begin{array}{l}\text { Compound heterozygous mutation } \\
\text { (Large deletion plus point mutation) }\end{array}$ & $4(19 \%)$ \\
Single exon deletion & $2(10 \%)$ \\
Point mutation & $5(24 \%)$ \\
Total & 21 \\
\hline
\end{tabular}

Family 8 is identical to family ARH11, which was reported in [1,2]. This family is only counted once in this table.

region. CNVs are genomic deletions or duplications greater than $1000 \mathrm{bp}$; smaller structural variations less than $1000 \mathrm{bp}$ are usually described as insertions or deletions (indels). Rapid improvements in genomic microarray technology have revealed an abundance of such structural variants in the human genome. In particular, human CNVs are significantly overrepresented close to telomeres, centromeres, and simple repeat regions. Furthermore, CNVs are enriched in genes of the immune and olfactory systems [20]. Thirty-two CNVs and four indels have so far been reported in the DOCK8 region (9p24.3) (http://projects.tcag.ca/variation), and proximity to the telomere could contribute to the large deletions often observed in DIDS patients [21].

The example of DIDS supports the hypothesis that CNV hotspots can contribute disproportionately to mutations in certain primary immunodeficiency diseases (PIDs). Previously, most studies focused on CNVs associated with common diseases [22-24]. Little attention was paid to structural variants as a marker for Mendelian diseases. We now know that about $12 \%$ of the human genome contains CNVs [25], and they increasingly rival single nucleotide polymorphisms (SNPs) in contributing to differences between individual human genomes [26]. Additionally, large deletions have been observed in X-linked agammaglobulinemia (XLA) [27] and severe combined immunodeficiency (SCID) [28,29]. These instances occur relatively infrequently compared to point mutations and small insertions/deletions, suggesting a previous underestimation of large deletions as a cause of known PIDs. Therefore, genome wide detection of CNVs using comparative genomic hybridization (CGH) arrays and SNP arrays could aid in mutational discovery and in diagnosis of both novel and known PIDs.

To provide additional insight into how these variants form, we sequenced genomic DNA spanning the deleted region in two patients who have large homozygous deletions (patient 1-1 and patient 2-1) [1]. In each patient, we found that the ends of the break- 
Table 2

Major clinical characteristics of DIDS

\begin{tabular}{|c|c|c|c|}
\hline Manifestations & & Zhang et al. [1] & Engelhardt et al. [2] \\
\hline Atopic dermatitis & & $100 \%$ & $95 \%$ \\
\hline Allergies & & $82 \%$ & $48 \%$ \\
\hline Skin and soft tissue infections & & $82 \%$ & $81 \%$ \\
\hline Sinopulmonary infections & & $91 \%$ & $100 \%$ \\
\hline Candida infections & & $45 \%$ & $81 \%$ \\
\hline \multirow[t]{5}{*}{ Cutaneous viral infections } & & $100 \%$ & $71 \%$ \\
\hline & Herpes Simplex Virus & $64 \%$ & $48 \%$ \\
\hline & Human Papillloma Virus & $64 \%$ & $14 \%$ \\
\hline & Molluscum Contagiosum & $45 \%$ & $33 \%$ \\
\hline & Others & $36 \%$ & $14 \%$ \\
\hline Malignancy & & $36 \%$ & $10 \%$ \\
\hline
\end{tabular}

point derive from a repeated small homologous region four to seven base pairs long. These findings support microhomology-mediated break-induced replication in generating large deletions in DOCK8 [30]. Indeed, although considered to occur infrequently, the same process is thought to underlie the origin of common or pathogenic CNVs in humans [21,25,26,31-35].

\subsection{Other DOCK8 mutations in DIDS}

Several DIDS patients have mutations that alter splice sites or introduce premature stop mutations [1,2]. These mutations typically exhibit nonsense-mediated decay, resulting in DOCK8 deficiency. Missense mutations that impair enzyme function by disrupting DHR1 or DHR2 could also mediate loss of function even if the full-length protein were preserved; however, no such mutations have yet been reported. At present, the most informative approach for detecting $D O C K 8$ mutations combines sequencing with expression level assays such as quantitative reverse-transcription polymerase chain reaction (qRT-PCR) or western blotting.

\section{Clinical and laboratory markers of DIDS}

\subsection{DIDS has a unique combination of clinical markers}

The clinical features of the 36 reported DIDS patients include recurrent infections, allergies, and malignancies. The clinical features and laboratory markers are summarized in Tables 2 and 3, respectively. To avoid any diagnostic uncertainty, in the rest of this review we discuss only the 30 patients who have proven genetic mutations on both alleles.

\subsection{Atopic dermatitis, allergy, and hyper-IgE}

Nearly all DIDS patients have atopic dermatitis (AD)(Table 2) [1,2]. AD affects about 15 to $30 \%$ of children and 2 to $10 \%$ of adults in the general population [36]. Genome-wide scans have identified multiple associated loci, including 5q31-q33 [37-41]. This locus, which contains the IL-4, IL-5, IL-12, and IL-13 genes, was also identified in a study of asthma and increased serum IgE [42]. In AD, allergen-specific CD4 $\mathrm{T}$ cells infiltrate the affected skin. The cells initially produce T helper type 2 (Th2) cytokines (IL-4, IL-5, IL-13), but later produce T helper type 1 (Th1) cytokines (IFN- $\gamma$ ) when the disease becomes chronic [38, $43,44]$. Although the above studies indicate that genetic susceptibility to and pathogenesis of AD is complex, patients having either DIDS or Wiskott-Aldrich syndrome (WAS) due to single gene mutations can also develop AD.

Since AD is usually observed before other symptoms develop, in males, DIDS should be distinguished from the X-linked disorder WAS. WAS presents with eczema, immunodeficiency, and bloody diarrhea due to thrombocytopenia [45]. Patients also display elevated IgE, Th2 skewing, and deficient suppressive function mediated by $\mathrm{T}$ regulatory $\left(\mathrm{T}_{\text {reg }}\right)$ cells [46]. The WAS protein interacts with CDC42 and the Arp2/3 complex to regulate the actin cytoskeleton $[45,47,48]$. Because DOCK 8 binds to CDC42 [15], DOCK8 may participate in the same signaling pathway, thereby explaining the clinical overlap between the two conditions.

All the described DIDS patients have elevated serum IgE (Table 3) [1,2], which might reflect an ascertainment bias, as patients were recruited from several HIES cohorts [49-53]. High serum IgE is also observed in other PIDs characterized by $\mathrm{T}$ cell dysfunction. These include autosomal dominant hyper-IgE 
Table 3

Immunological laboratory findings in DIDS

\begin{tabular}{llll}
\hline Laboratory tests & & Zhang et al. [1] & Engelhardt et al. [2] \\
\hline Lymphopenia & All T cells & $90 \%$ & $38 \%$ \\
& CD4 T cells & $100 \%$ & $54 \%$ \\
& CD8 T cells & $90 \%$ & $25 \%$ \\
B cell counts & & $36 \%$ low & $0 \%$ low \\
NK cell counts & & $60 \%$ low & $13 \%$ low \\
Eosinophilia & & $100 \%$ & $100 \%$ \\
Immunoglobulins & IgG & $100 \%$ normal to high & $100 \%$ normal to high \\
& IgA & $18 \%$ low & $19 \%$ low \\
& & $82 \%$ normal to high & $81 \%$ normal to high \\
& IgE & $100 \%$ high & $100 \%$ high \\
& IgM & $100 \%$ low & $77 \%$ low \\
\hline
\end{tabular}

Two patients were reported in both papers (Patient 8-1 and Patient 8-2 as ARH11.4 and ARH11.5, respectively). These patients were included in each of the calculations for both series $[1,2]$.

syndrome (AD-HIES) due to STAT3 mutations, DiGeorge syndrome, severe combined immunodeficiency disease (SCID) variants such as Omenn syndrome, and WAS. In DIDS, eosinophilia often accompanies the high IgE levels (Table 3) [1,2], and eosinophilic esophagitis or eosinophilic pneumonitis can occur [1]. Unlike the other disorders, in which high IgE has unclear clinical consequences, DIDS patients often exhibit allergies to multiple food and environmental allergens, as well as asthma (Table 2) [1,2]. The mechanism responsible for allergy and hyper IgE in DIDS has not yet been established. Because skewed Th2 responses are a hallmark for allergy, the Th1/Th2 responses in these patients warrants testing.

\subsection{Skin and soft tissue infections}

DIDS patients are susceptible to bacterial infections involving the skin and soft tissues. Most patients develop recurrent skin abscesses, cellulitis, and otitis externa (Table 2) [1,2]. Staphylococcus aureus is the most frequent pathogen, but Acinetobacter baumanii has also been reported [1,2]. Patients can also develop Candida infections of the skin, nails, as well as oral and vaginal mucosal surfaces [1,2]. Engelhardt et al. reported candidiasis in $81 \%$ of their patients, who were predominantly Turkish [2]. However, our North American cohort had Candida infections in only $45 \%$ of the patients (Table 2) [1], which may reflect different genetic backgrounds, antibiotic usage, or other environmental conditions $[54,55]$. While chronic atopic dermatitis may impair skin barrier function and predispose to secondary bacterial superinfections, it is also possible that DOCK8 has an intrinsic role in skin defense.

Bacterial infections involving the skin and soft tissues, as well as candidiasis, are prominent features of
STAT3 mutant AD-HIES [56]. The infectious susceptibility in AD-HIES patients can be explained by their lack of, and inability to generate, CD4 T helper type 17 (Th17) cells, which contribute to defense against extracellular bacteria and fungi [51]. HIES-like patients, who were included as controls in this study, showed an intermediately decreased percentage of Th17 cells. The HIES-like group is now known to have included two DIDS patients (patients 14 and 15 in [51] are patients 4-1 and 4-2, respectively, in [1]). These results were extended in a second study that compared AD-HIES patients (HIES-STAT3mut) to HIES patients who lacked STAT3 mutations (HIES-STAT3wt) [57]. $\mathrm{T}$ cells from both patient groups had decreased expression of IL-17 and the ROR $\gamma \mathrm{t}$ transcription factor required for Th17 differentiation. Confirming the earlier study, HIES-STAT3mut patients were blocked in their ability to generate Th17 cells from naïve T cells. However, naive T cells from HIES-STAT3wt patients, when differentiated under Th17 conditions, produced less IL-17 despite expressing normal levels of ROR $\gamma \mathrm{t}$. The HIES-STAT3wt group is now known to have included six DIDS patients (patients P14, P19, P17, P7, P11, P2 in [57] are patients ARH001, AR002, AR003, ARH004, ARH008, ARH009, respectively, in [2]). Together, these two studies suggest that DIDS patients have a mild to moderate defect at the later stages of Th17 differentiation or affecting survival of Th17 cells. This finding could explain what would appear to be a milder degree of increased susceptibility to Candida infections in DIDS as compared to AD-HIES patients.

\subsection{Recurrent respiratory tract infections}

Most DIDS patients have recurrent or chronic upper and lower respiratory tract infections (Table 2) [1,2]. 
Specifically, many patients require sinus surgery and myringotomy tube placement for sinusitis and otitis media, and some develop mastoiditis. Most patients also have recurrent pneumonias and bronchitis, and some develop bronchiectasis. Pulmonary pathogens that have been identified include Streptococcus pneumoniae, Haemophilus influenzae, Pneumocystis jirovecii, respiratory adenovirus, and respiratory syncytial virus. Some patients also have Giardia or Salmonella infections of the gastrointestinal tract [1,2].

In DIDS patients, the high frequency of recurrent infections in the respiratory and gastrointestinal tracts and their improvement after starting long-term replacement of immunoglobulins - suggests defects in humoral immunity [1]. However, a discrepancy exists between specific antibody production and total immunoglobulin levels in the DIDS cohorts described thus far. Unlike patients with XLA or common variable immunodeficiency (CVID), DIDS patients usually have normal or even increased serum $\mathrm{IgG}$ and $\mathrm{IgA}$ (Table 3) [1,2]. IgA, which is especially important for mucosal immunity, is low in only a minority. These immunoglobulin levels bear no relationship to circulating B cell numbers, which in some cases can be mildly decreased (Table 3) [1,2]. By contrast, patients have impaired antibody responses to vaccines, including both $\mathrm{T}$ cell-dependent antigens found in protein conjugate vaccines and $T$ cell-independent antigens such as in the pneumococcal polysaccharide vaccine [1]. The impairment in antibody responses is highly variable, differing for the individual patient, the specific antigen, and in degree of impairment [1]. An inability to sustain effective antibody responses after secondary challenge is also observed in Dock8-deficient mice [3]. Therefore, the inadequate titers of antibodies to specific pathogens are most likely responsible for the recurrent respiratory tract infections seen in DIDS, regardless of total isotype levels or B cell numbers.

Further complicating the picture, IgM is decreased in most DIDS patients (Table 3) [1,2]. After initial activation by antigen, the responding $\mathrm{B}$ cells migrate into germinal centers where they undergo somatic hypermutation and isotype class switch recombination with the help of $\mathrm{T}$ cells. This latter process results in the responding B cells "switching" from IgM production to $\operatorname{IgG}, \operatorname{IgA}$, and $\operatorname{IgE}$ production. It is unclear how DIDS patients develop hypo-IgM and hyper-IgE. The initial activation signal through the $\mathrm{B}$ cell receptor, the directed migration of B cells inside lymph nodes, and the second signals provided by $\mathrm{T}$ cells can each affect the final production of certain isotypes [58]. Lack of DOCK8 at any of these steps could contribute to the observed abnormalities in antibody production.

\subsection{Cutaneous viral infections}

Severe or chronic cutaneous viral infections constitute a distinctive feature in DIDS (Table 2) [1,2]. Herpes simplex virus (HSV), human papilloma virus (HPV), molluscum contagiosum virus (MCV), and varicella-zoster virus infections of the skin are common. Co-infections occur in most patients. These DNA viruses are widespread, transferred by skin contact, and cause limited infections in healthy people. However, in DIDS, such infections remain difficult to control, even when patients receive biological modifiers such as IFN- $\alpha$. By contrast, systemic viral infections rarely occur in DIDS. Although a few cases of JC virus of the brain and one case of cytomegalovirus viremia were observed [2], no cases of HSV encephalitis or systemic Epstein-Barr virus infection have been reported.

Susceptibility to viral infections, including skin infections with HSV, HPV, and MCV, occur in PIDs such as WAS and "leaky" SCID [59-61]. These disorders highlight the importance of $\mathrm{T}$ cells in defense against viral infections. One PID that shares a selective susceptibility to cutaneous viral infections is the WHIM syndrome (Warts, Hypogammaglobulinemia, Infections, and Myelokathexis syndrome). WHIM syndrome is caused by mutations in CXCR4, a chemokine receptor that regulates leukocyte migration into tissues [62-64]. The chemokines that activate CXCR4 are expressed in multiple organs, especially in different infections and under inflammatory conditions [65-67]. Thus, control of certain viral infections in the skin requires effective migration of leukocytes into the infected tissue.

To better understand why DIDS presents with increased susceptibility to viral infections, we assessed CD8 T cell functions, which are critical for antiviral defense [1]. DIDS patients demonstrate defective CD8 $\mathrm{T}$ cell expansion in response to TCR stimulation with anti-CD3 plus anti-CD28 antibodies. CD8 T cell production of antiviral cytokines such as TNF- $\alpha$ and IFN$\gamma$ are partially decreased. However, cytotoxicity of cycling CD8 T cells appears intact, as indicated by in vitro assays showing normal cytotoxic granule exocytosis and perforin content [1]. Given the documented chemotactic abnormalities in Dock2-deficient mice [17,18], similar abnormalities in DOCK8-deficient cells are expected. Therefore, in DIDS the increased susceptibility to viral infections could result from a combination of factors: defective barrier function of eczematous skin, decreased peripheral T cells, impaired CD8 T cell expansion, decreased production of antiviral cytokines, and inhibited cell migration into infected skin. 


\subsection{Malignancy}

DIDS patients are at increased risk of developing squamous cell carcinoma and lymphoid malignancies (Table 2) [1,2]. These malignancies could result from chronic HPV infections and defective T cell-mediated immune surveillance. Alternatively, DOCK8 could normally exert an intrinsic tumor suppressor role, so that its loss in DIDS patients could contribute to carcinogenesis. Indeed, complex structural rearrangements characterized by loss of chromosome $9 p$ have long been associated with malignancy [68]. Furthermore, homozygous 9p24 deletions, which encompass the DOCK 8 gene, have been observed in human lung, gastric, and pancreatic carcinoma cell lines [69-72] Gliomas also acquire DOCK8 deletions during their progression from primary to higher-grade malignancies [73]. These and other studies have shown that DOCK 8 is abnormally regulated in different human malignancies. Whereas DOCK8 expression was increased in a radiosensitive esophageal cancer cell line compared to radioresistant lines [74], its expression is decreased in a large number of primary hepatocellular [75] and lung cancers [69]. Overall, the studies in different human malignancies suggest that loss of DOCK8 expression in non-immune cells contributes to carcinogenesis. If so, then hematopoietic cell transplantation, which is curative in many PIDs, may not fully prevent the development of malignancies in DIDS patients.

\subsection{T cell lymphopenia}

DIDS patients display a progressive lymphopenia (Table 3) [1,2]. The decrease prominently affects $\mathrm{T}$ cells, including both CD4 and CD8 T cell subsets (Table 3) $[1,2]$. However, some patients also have decreased NK cell and/or B cell numbers $[1,2]$. Decreased lymphocyte numbers and defective lymphocyte function define this disorder as a combined immunodeficiency $[1,2]$.

How DOCK8-deficiency causes T cell lymphopenia is still incompletely understood. We previously showed that in vitro TCR stimulation leads to impaired activation, cell division, and expansion of $\mathrm{T}$ cells, especially of CD8 T cells, in peripheral blood mononuclear leukocytes from DIDS patients [1]. The decreased T proliferation was also confirmed by Engelhardt et al. in additional patients [2]. These findings are consistent with work showing that the Rho-GTPases (especially RAC1, RAC2, and CDC42), through which DOCK8 likely acts, are important for T cell activation, proliferation, and survival $[9,11,76]$. Whether the T cell impairment results from defective TCR signaling, insufficient cytokine production, or impaired cell cycle progression remains to be determined.

Similar to DIDS patients, Dock8-deficient mice have decreased $\mathrm{T}$ cell numbers, including both CD4 and CD8 T cells and especially affecting naïve CD4 T cells (see below) [3]. Bone marrow chimeras were generated by adoptively transferring Dock8-deficient mutant cells mixed with equal numbers of wild-type cells into irradiated mice. Reconstitution resulted in only $5 \%$ mutant $\mathrm{T}$ cells of the total peripheral $\mathrm{T}$ cells, suggesting that mutant cells are at a competitive disadvantage [3].

The $\mathrm{T}$ cell lymphopenia in DOCK8 deficient humans and mice could also result from defective $\mathrm{T}$ cell development in the thymus, insufficient thymic egress, or abnormal migration of mature $\mathrm{T}$ cells. In addition to being expressed in mature peripheral $\mathrm{T}$ cells [1], DOCK8 is expressed in hematopoietic stem cells (http://refdic.rcai.riken.jp/welcome.cgi) and thymocytes [77]. Thymic development is blocked to varying degrees in mice who have deficient or impaired Rho GTPases (Rac1/Rac2 or Cdc42), or who lack Vav1, a GEF upstream of the Rho GTPases [78-82]. The Rho-GTPases, especially Rac1, Cdc42, and RhoA, play a key role in leukocyte adhesion and chemokinedirected migration $[6,11,83,84]$. Perturbations in migration could impair thymic egress, as well as alter in vivo trafficking patterns of mature T cells. To help distinguish among these possibilities, it will be informative to examine TCR excision circles (TRECs) or other markers of recent thymic emigrants, as well as enumerate $\mathrm{T}$ cells in lymphoid organs and non-immune tissues in mice.

\section{Dock8-deficient mouse models}

Randall et al. recently reported two lines of Dock8deficient mice, which were independently generated by N-ethyl-N-nitrosourea mutagenesis [3]. The mice were identified in a B cell functional screen by their failure to generate a mature and persistent secondary antibody response. This failure results from defects in both survival of germinal center B cells and ICAM1 accumulation into the immunological synapse of $\mathrm{B}$ cells. The mice also exhibit lower $\mathrm{T}$ cell counts and $\mathrm{T}$ cell-dependent antibody responses [3].

The Dock8-deficient mice therefore recapitulate several essential findings observed in the DIDS patients, 
namely the lack of memory response observed in DIDS patients undergoing secondary challenge with bacteriophage $\phi 174$ or other antigens, and their T cell lymphopenia [1]. It will be interesting to challenge these mice with allergens, chronic viral infections, or cancerpromoting agents. The fortuitous discovery of the mice will make it possible to establish disease models that more closely resemble the human condition, as well as facilitate more detailed mechanistic studies of this fascinating human disease.

\section{Conclusion}

In summary, DIDS is a newly described combined immunodeficiency caused by autosomal recessive, loss-of-function mutations in DOCK8. Clinical and laboratory markers of DIDS include cutaneous viral infections, allergies, malignancies, lymphopenia, decreased IgM, increased IgE, and eosinophilia. Patients often have large homozygous or compound heterozygous deletions, although point mutations and splice site mutations can be identified. Genetic techniques that efficiently measure large deletions should complement traditional exonic sequencing for molecular diagnosis. Investigations in DIDS patients and their counterparts in mice implicate both $\mathrm{T}$ cell and $\mathrm{B}$ cell abnormalities in disease pathogenesis. Further mechanistic studies will help us understand the role of this poorly understood GEF in normal lymphocyte function, as it pertains to control of allergy, viral infections, and cancer in the general population.

Note in proof: While in press, Gatz et al. [85] reported two new DIDS patients who were cured by hemopoietic cell transplantation.

\section{Acknowledgements}

This work was supported by the Intramural Research Program of the National Institutes of Health, the National Institute of Allergy and Infectious Diseases. J.C.D also received support from the Lazarus Family Scholarship through the George Washington University School of Medicine and Health Sciences. We thank Huie Jing for sharing unpublished data; Yu Zhang for helpful discussions; and Andrew Snow, Dara StraussAlbee, and Bernice Lo for reading this manuscript.

\section{References}

[1] Q. Zhang et al., Combined immunodeficiency associated with DOCK8 mutations, N Engl J Med 361 (2009), 2046-2055.

[2] K.R. Engelhardt et al., Large deletions and point mutations involving the dedicator of cytokinesis 8 (DOCK8) in the autosomal-recessive form of hyper-IgE syndrome, J Allergy Clin Immunol 124 (2009), 1289-302 e4.

[3] K.L. Randall et al., Dock8 mutations cripple B cell immunological synapses, germinal centers and long-lived antibody production, Nat Immunol 10 (2009), 1283-1291.

[4] J.F. Cote and K. Vuori, Identification of an evolutionarily conserved superfamily of DOCK180-related proteins with guanine nucleotide exchange activity, J Cell Sci 115 (2002), 49014913.

[5] N. Meller, S. Merlot and C. Guda, CZH proteins: a new family of Rho-GEFs, J Cell Sci 118 (2005), 4937-4946.

[6] K.L. Rossman, C.J. Der and J. Sondek, GEF means go: turning on RHO GTPases with guanine nucleotide-exchange factors, Nat Rev Mol Cell Biol 6 (2005), 167-180.

[7] M. Lu and K.S. Ravichandran, Dock180-ELMO cooperation in Rac activation, Methods Enzymol 406 (2006), 388-402.

[8] Y. Miyamoto et al., Dock6, a Dock-C subfamily guanine nucleotide exchanger, has the dual specificity for Rac 1 and $\mathrm{Cdc} 42$ and regulates neurite outgrowth, Exp Cell Res 313 (2007), 791-804.

[9] S. Etienne-Manneville and A. Hall, Rho GTPases in cell biology, Nature 420 (2002), 629-635.

[10] S.J. Heasman and A.J. Ridley, Mammalian Rho GTPases: new insights into their functions from in vivo studies, Nat Rev Mol Cell Biol 9 (2008), 690-701.

[11] V.L. Tybulewicz and R.B. Henderson, Rho family GTPases and their regulators in lymphocytes, Nat Rev Immunol 9 (2009), 630-644.

[12] K. Wennerberg, K.L. Rossman and C.J. Der, The Ras superfamily at a glance, J Cell Sci 118 (2005), 843-846.

[13] J. Yamauchi et al., ErbB2 directly activates the exchange factor Dock7 to promote Schwann cell migration, J Cell Biol 181 (2008), 351-365.

[14] M. Watabe-Uchida et al., The Rac activator DOCK7 regulates neuronal polarity through local phosphorylation of stathmin/Op18, Neuron 51 (2006), 727-739.

[15] A. Ruusala and P. Aspenstrom, Isolation and characterisation of DOCK8, a member of the DOCK180-related regulators of cell morphology, FEBS Lett 572 (2004), 159-166.

[16] A.L. Blasius et al., Mice with mutations of Dock7 have generalized hypopigmentation and white-spotting but show normal neurological function, Proc Natl Acad Sci U S A 106 (2009), 2706-2711.

[17] Y. Fukui et al., Haematopoietic cell-specific CDM family protein DOCK2 is essential for lymphocyte migration, Nature 412 (2001), 826-831.

[18] Y. Kunisaki et al., DOCK2 is a Rac activator that regulates motility and polarity during neutrophil chemotaxis, J Cell Biol 174 (2006), 647-652.

[19] T. Sanui et al., DOCK2 is essential for antigen-induced translocation of TCR and lipid rafts, but not PKC-theta and LFA-1, in T cells, Immunity 19 (2003), 119-129.

[20] D.Q. Nguyen, C. Webber and C.P. Ponting, Bias of selection on human copy-number variants, PLoS Genet 2 (2006), e20.

[21] P.J. Hastings et al., Mechanisms of change in gene copy number, Nat Rev Genet 10 (2009), 551-564. 
[22] T.J. Aitman et al., Copy number polymorphism in Fcgr3 predisposes to glomerulonephritis in rats and humans, Nature 439 (2006), 851-855

[23] E.G. Bochukova et al., Large, rare chromosomal deletions associated with severe early-onset obesity, Nature 463 (2008), 666-670.

[24] E.H. Cook, Jr. and S.W. Scherer, Copy-number variations associated with neuropsychiatric conditions, Nature 455 (2008), 919-923.

[25] R. Redon et al., Global variation in copy number in the human genome, Nature 444 (2006), 444-454.

[26] S.A. McCarroll et al., Integrated detection and populationgenetic analysis of SNPs and copy number variation, Nat Genet 40 (2008), 1166-1174.

[27] J. Rohrer et al., Unusual mutations in Btk: an insertion, a duplication, an inversion, and four large deletions, Clin Immunol 90 (1999), 28-37.

[28] M.L. Markert et al., Adenosine deaminase (ADA) deficiency due to deletion of the ADA gene promoter and first exon by homologous recombination between two Alu elements, J Clin Invest 81 (1988), 1323-1327.

[29] T.M. Berkvens et al., Identical 3250-bp deletion between two AluI repeats in the ADA genes of unrelated ADA-SCID patients, Genomics 7 (1990), 486-490.

[30] J.O. Korbel et al., Paired-end mapping reveals extensive structural variation in the human genome, Science 318 (2007), 420-426.

[31] P.J. Hastings, G. Ira and J.R. Lupski, A microhomologymediated break-induced replication model for the origin of human copy number variation, PLoS Genet 5 (2009), e1000327.

[32] G.M. Cooper, D.A. Nickerson and E.E. Eichler, Mutational and selective effects on copy-number variants in the human genome, Nat Genet 39 (2007), S22-S29.

[33] M. Jakobsson et al., Genotype, haplotype and copy-number variation in worldwide human populations, Nature 451 (2008), 998-1003.

[34] J. Sebat et al., Large-scale copy number polymorphism in the human genome, Science 305 (2004), 525-528.

[35] L.E. Vissers et al., Rare pathogenic microdeletions and tandem duplications are microhomology-mediated and stimulated by local genomic architecture, Hum Mol Genet 18 (2009), 35793593.

[36] H. Williams and C. Flohr, How epidemiology has challenged 3 prevailing concepts about atopic dermatitis, J Allergy Clin Immunol 118 (2006), 209-213.

[37] W.O. Cookson et al., Genetic linkage of childhood atopic dermatitis to psoriasis susceptibility loci, Nat Genet 27 (2001), 372-373.

[38] T. Bieber, Atopic dermatitis, N Engl J Med 358 (2008), 148394.

[39] L.J. Palmer and L.R. Cardon, Shaking the tree: mapping complex disease genes with linkage disequilibrium, Lancet 366 (2005), 1223-1234.

[40] Y.A. Lee et al., A major susceptibility locus for atopic dermatitis maps to chromosome 3q21, Nat Genet 26 (2000), 470-473.

[41] A. Haagerup et al., Atopic dermatitis - a total genome-scan for susceptibility genes, Acta Derm Venereol 84 (2004), 346-352.

[42] D.S. Postma et al., Genetic susceptibility to asthma-bronchial hyperresponsiveness coinherited with a major gene for atopy, N Engl J Med 333 (1995), 894-900.

[43] M. Grewe et al., Analysis of the cytokine pattern expressed in situ in inhalant allergen patch test reactions of atopic dermatitis patients, J Invest Dermatol 105 (1995), 407-410.
[44] R.A. Taha et al., In vivo expression of cytokine receptor mRNA in atopic dermatitis, J Allergy Clin Immunol 102 (1998), 245250.

[45] G. Bouma, S.O. Burns and A.J. Thrasher, Wiskott-Aldrich Syndrome: Immunodeficiency resulting from defective cell migration and impaired immunostimulatory activation, Immunobiology 214 (2009), 778-790.

[46] E. Ozcan, L.D. Notarangelo and R.S. Geha, Primary immune deficiencies with aberrant IgE production, J Allergy Clin Immunol 122 (2008), 1054-1062; quiz 1063-1064.

[47] R. Guinamard et al., Tyrosine phosphorylation of the WiskottAldrich syndrome protein by Lyn and Btk is regulated by CDC42, FEBS Lett 434 (1998), 431-436.

[48] P. Aspenstrom, U. Lindberg and A. Hall, Two GTPases, Cdc42 and Rac, bind directly to a protein implicated in the immunodeficiency disorder Wiskott-Aldrich syndrome, Curr Biol 6 (1996), 70-75.

[49] A.F. Freeman and S.M. Holland, Clinical manifestations, etiology, and pathogenesis of the hyper-IgE syndromes, Pediatr Res 65 (2009), 32R-37R.

[50] E.D. Renner et al., Autosomal recessive hyperimmunoglobulin E syndrome: a distinct disease entity, J Pediatr 144 (2004), 93-99.

[51] J.D. Milner et al., Impaired T(H)17 cell differentiation in subjects with autosomal dominant hyper-IgE syndrome, Nature 452 (2008), 773-776

[52] J.Y. Lei et al., Microcystic adnexal carcinoma associated with primary immunodeficiency, recurrent diffuse herpes simplex virus infection, and cutaneous T-cell lymphoma, Am J Dermatopathol 22 (2000), 524-529.

[53] D. Pfeifer et al., The hyper-IgE syndrome is not caused by a microdeletion syndrome, Immunogenetics 59 (2007), 913926.

[54] H. Yapicioglu et al., A 6-year prospective surveillance of healthcare-associated infections in a neonatal intensive care unit from southern part of Turkey, J Paediatr Child Health.

[55] E.G. Playford et al., Increasing incidence of candidaemia: long-term epidemiological trends, Queensland, Australia, 1999-2008, J Hosp Infect.

[56] S.M. Holland et al., STAT3 mutations in the hyper-IgE syndrome, N Engl J Med 357 (2007), 1608-1619.

[57] S. Al Khatib et al., Defects along the $\mathrm{T}(\mathrm{H}) 17$ differentiation pathway underlie genetically distinct forms of the hyper $\operatorname{IgE}$ syndrome, J Allergy Clin Immunol 124 (2009), 342-348, 348 e1-e5.

[58] L.D. Notarangelo et al., Defects of class-switch recombination, J Allergy Clin Immunol 117 (2006), 855-864.

[59] M. Saijo et al., Recurrent aciclovir-resistant herpes simplex in a child with Wiskott-Aldrich syndrome, Br J Dermatol 139 (1998), 311-314.

[60] H. Artac et al., Late-onset adenosine deaminase deficiency presenting with Heck's disease, Eur J Pediatr 169, 1033-1036.

[61] P. Modiano et al., Acyclovir-resistant chronic cutaneous herpes simplex in Wiskott-Aldrich syndrome, Br J Dermatol 133 (1995), 475-478.

[62] B. Lagane et al., CXCR4 dimerization and beta-arrestinmediated signaling account for the enhanced chemotaxis to CXCL12 in WHIM syndrome, Blood 112 (2008), 34-44.

[63] P.A. Hernandez et al., Mutations in the chemokine receptor gene CXCR4 are associated with WHIM syndrome, a combined immunodeficiency disease, Nat Genet 34 (2003), 70-74.

[64] K. Balabanian et al., WHIM syndromes with different genetic anomalies are accounted for by impaired CXCR4 desensitization to CXCL12, Blood 105 (2005), 2449-2457. 
[65] K. Tashiro et al., Signal sequence trap: a cloning strategy for secreted proteins and type I membrane proteins, Science $\mathbf{2 6 1}$ (1993), 600-603.

[66] J.A. Gonzalo et al., Critical involvement of the chemotactic axis CXCR4/stromal cell-derived factor-1 alpha in the inflammatory component of allergic airway disease, J Immunol 165 (2000), 499-508.

[67] W.J. Cook et al., Persistent expression of chemokine and chemokine receptor RNAs at primary and latent sites of herpes simplex virus 1 infection, Virol J1 (2004), 5.

[68] M. Sato et al., Identification of chromosome arm $9 p$ as the most frequent target of homozygous deletions in lung cancer, Genes Chromosomes Cancer 44 (2005), 405-414.

[69] K. Takahashi et al., Homozygous deletion and reduced expression of the DOCK8 gene in human lung cancer, Int J Oncol 28 (2006), 321-328.

[70] M. Heidenblad et al., Genome-wide array-based comparative genomic hybridization reveals multiple amplification targets and novel homozygous deletions in pancreatic carcinoma cell lines, Cancer Res 64 (2004), 3052-3059.

[71] J.U. Kang et al., Frequent silence of chromosome 9p, homozygous DOCK8, DMRT1 and DMRT3 deletion at 9p24.3 in squamous cell carcinoma of the lung, Int J Oncol 37, 327-35.

[72] H. Takada et al., Genomic loss and epigenetic silencing of very-low-density lipoprotein receptor involved in gastric carcinogenesis, Oncogene 25 (2006), 6554-6562.

[73] A. Idbaih et al., Genomic changes in progression of low-grade gliomas, J Neurooncol 90 (2008), 133-140.

[74] R. Ogawa et al., Identification of candidate genes involved in the radiosensitivity of esophageal cancer cells by microarray analysis, Dis Esophagus 21 (2008), 288-297.

[75] P. Saelee et al., Novel PNLIPRP3 and DOCK8 gene expression and prognostic implications of DNA loss on chromosome 10q25.3 in hepatocellular carcinoma, Asian Pac J Cancer Prev 10 (2009), 501-506.

[76] S. Sinha and W. Yang, Cellular signaling for activation of Rho GTPase Cdc42, Cell Signal 20 (2008), 1927-1934.

[77] M.S. Lee et al., Gene expression profiles during human CD4+ T cell differentiation, Int Immunol 16 (2004), 1109-1124.

[78] C. Dumont et al., Rac GTPases play critical roles in early T-cell development, Blood 113 (2009), 3990-3998.

[79] K. Smits et al., Rho GTPase Cdc42 is essential for human T-cell development, Haematologica 95, 367-375.

[80] A. Tarakhovsky et al., Defective antigen receptor-mediated proliferation of B and $\mathrm{T}$ cells in the absence of Vav, Nature 374 (1995), 467-470.

[81] K.D. Fischer et al., Defective T-cell receptor signalling and positive selection of Vav-deficient CD4+ CD8+ thymocytes, Nature 374 (1995), 474-477.

[82] R. Zhang et al., Defective signalling through the T- and Bcell antigen receptors in lymphoid cells lacking the vav protooncogene, Nature 374 (1995), 470-473.

[83] A. Mor, M.L. Dustin and M.R. Philips, Small GTPases and LFA-1 reciprocally modulate adhesion and signaling, Immunol Rev 218 (2007), 114-125.

[84] M. Machacek et al., Coordination of Rho GTPase activities during cell protrusion, Nature 461 (2009), 99-103.

[85] S.A. Gatz et al., Curative treatment of autosomal-recessive hyper-IgE syndrome by hematopoietic cell transplantation, Bone Marrow Transplant, (2010), [Epub ahead of print]. 


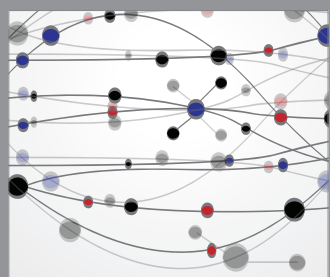

The Scientific World Journal
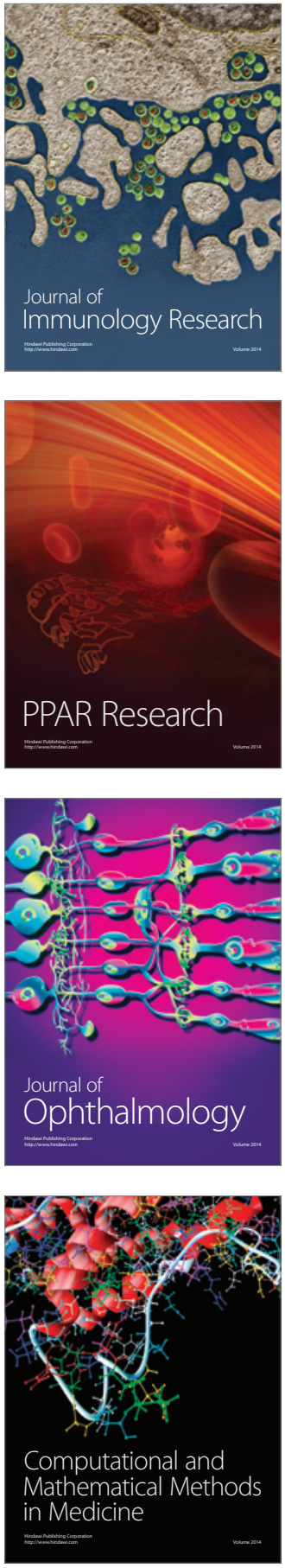

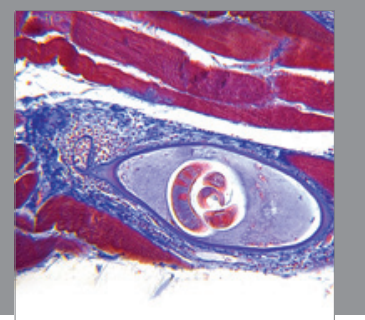

Gastroenterology

Research and Practice
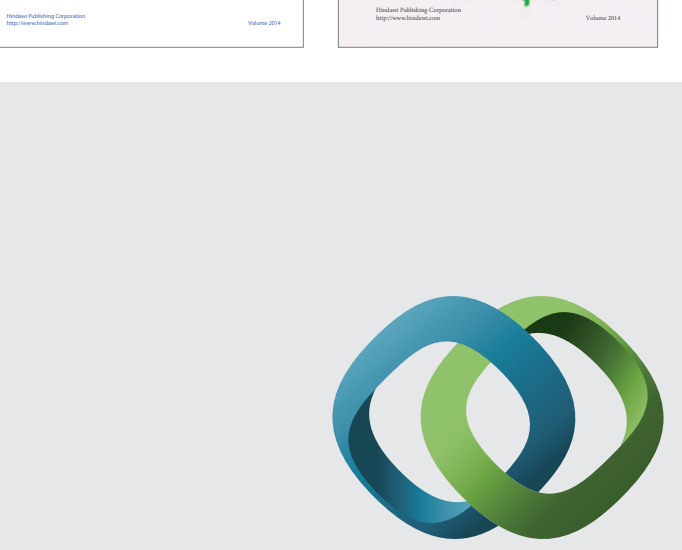

\section{Hindawi}

Submit your manuscripts at

http://www.hindawi.com
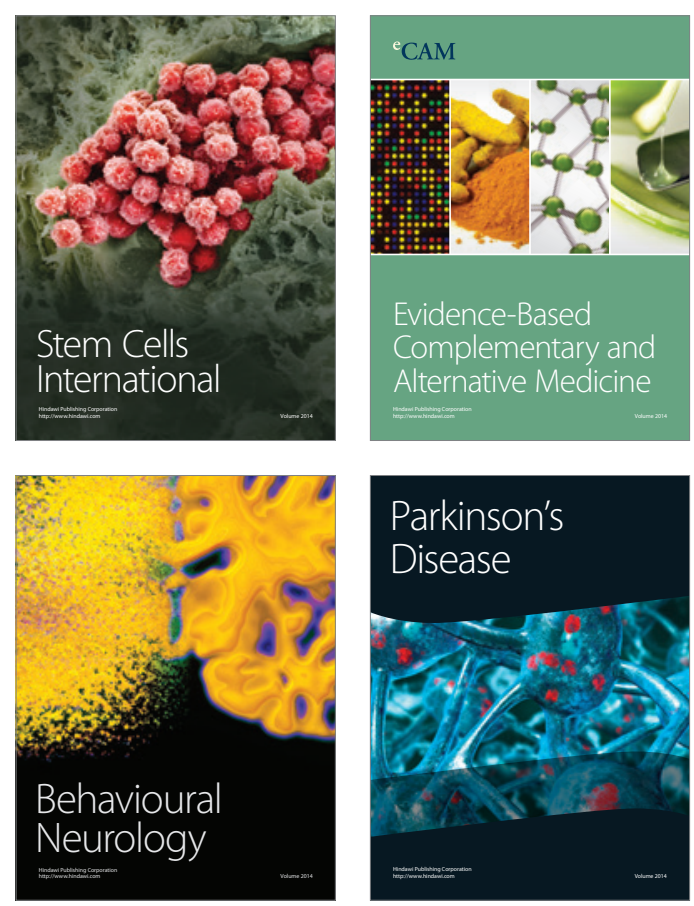

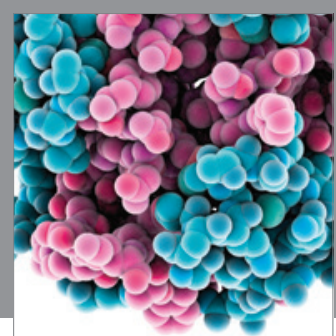

Journal of
Diabetes Research

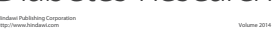

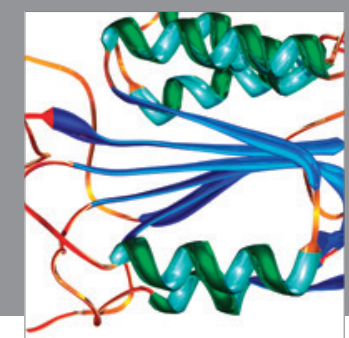

Disease Markers
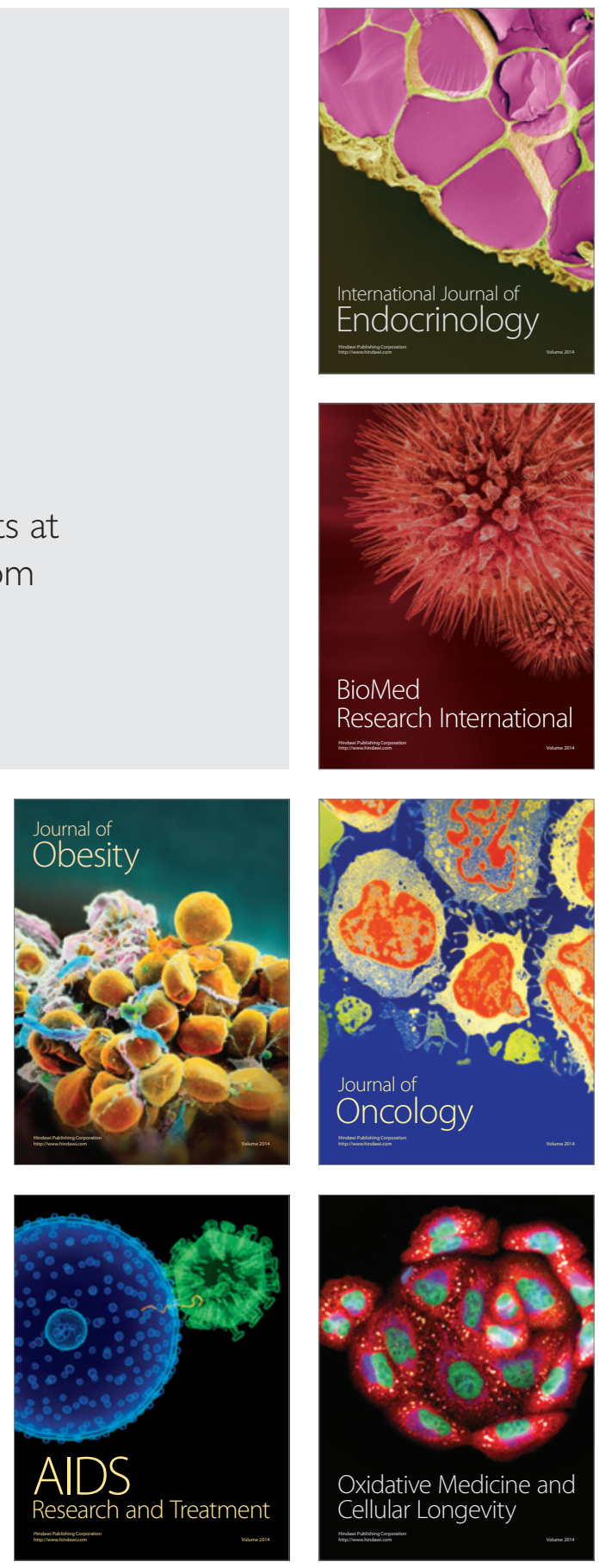